\title{
Workshop Report: Current Performance Testing for Pharmaceutical and TCM Dosage Forms
}

\author{
Raimer Löebenberg ${ }^{1}$, Hai Wei ${ }^{2}$, and Jianfang Feng ${ }^{2}$ \\ 1 University of Alberta, Edmonton AB, Canada \\ ${ }^{2}$ Shanghai University of Traditional Chinese Medicine, Shanghai, China
}

$\mathrm{O}$ n October 23-24, 2008, the Shanghai University of Traditional Chinese Medicine sponsored a workshop on the pressing issue of performance and quality benchmarks for conventional and traditional medicines. Dr. Gordon Amidon from the College of Pharmacy at the University of Michigan, who is the father of the Biopharmaceutical Classification System (BCS), gave a comprehensive overview of the current status and the future implication of the BCS for the pharmaceutical and traditional industry. The U.S. FDA uses the BCS for regulatory decisions. One of the most important uses of the BCS classification is to define which drug products may qualify for Biowaivers in the pre- and post-approval phases. The principles of the BCS can benefit traditional drugs by classifying their components. This will help to set meaningful quality control standards for botanical remedies. Dr. Erika Stippler, director of dosage form performance laboratory of the United States Pharmacopeia, spoke about the importance of the new USP performance verification test. She showed how the physical calibration of a dissolution apparatus can impact results. However, other factors like the laboratory environment and the degassing technique, together with the work style of analyst, might influence the results to an even higher degree. Dr. Raimar Löbenberg, University of Alberta, gave an overview of the current status of in vitro-in vivo correlation and its implications for traditional medicines. He showed how the knowledge and techniques used for pharmaceuticals can be applied to traditional medicines. Dissolution and permeability data can be used in computer simulations to establish IVIVC for herbal drugs. Bioequivalence of herbals is possible using these methods. Prof. Izadore Kanfer from South Africa gave examples of how dissolution methods can be developed for different herbal drugs. His lab developed an assay and a dissolution method for the African potato, a traditional plant with important health benefits for South Africans. Different products containing this herb from the South African Market were evaluated, and significant differences were found. Dr. Joan Z. Zuo, School of Pharmacy, The Chinese University of Hong Kong, talked about identification of relevant chemical markers for quality control of TCM products. Her example was Danshen, the dried root of Saliva miltiorrhiza Bge. In clinical studies, Fufang Danshen Dripping Pill was found to be as effective as sublingual nitroglycerin and isosorbide dinitrate. However, the available pharmacokinetic information is inconsistent. The BCS characterization of major components of this plant shows that they are permeability limited, which is in line with the reported variable absorption.

Bryan Crist, Varian Inc., wrapped up the workshop with an interesting talk about the basics of dissolution testing techniques. He gave an overview of the different apparatus and their uses. The workshop was concluded with a hands-on demonstration of dissolution equipment and instruction on performing a dissolution test according to current USP specifications.

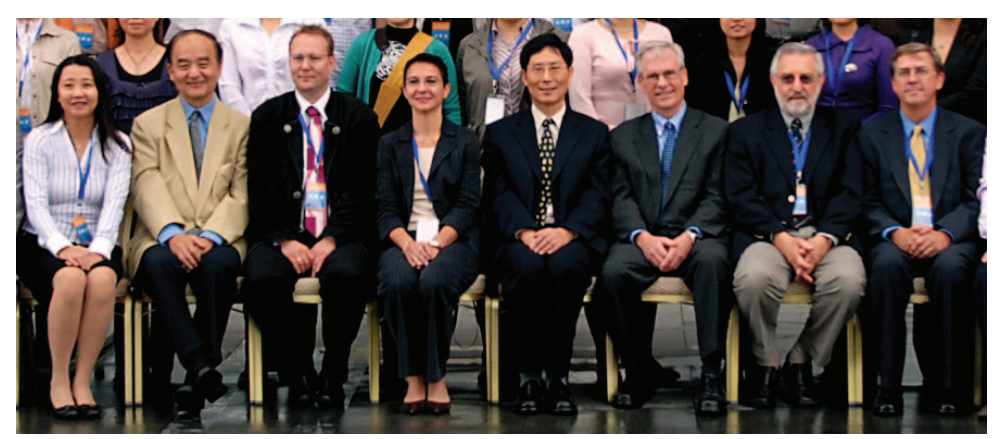

From left to right: Dr. Joan Zhong Zou, Prof. Qinghua Cheng, Dr. Raimar Löbenberg, Dr. Erika Stippler, Prof. Kaixian Cheng, Dr. Gordon Amidon, Prof. Isadore Kanfer, Mr. Bryan Crist 